\title{
Erreichbarkeitsgraphen als Werkzeug zur Visualisierung des Treibhausgasausstoßes für die Verkehrs mittel Flugzeug, Auto, Bahn und Reisebus bei der Dienstreiseplanung
}

\author{
Malte Christiansen ${ }^{1}$ J ochen W ittmann ${ }^{1}$ \\ ${ }^{1}$ Hochschule für Technik und Wirtschaft Berlin, Studiengang Umweltinformatik, \\ Wilhelminenhofstraße 75A, 12459 Berlin, Germany, wittmann@ htw-berlin.de
}

\begin{abstract}
Die Angestellten der HTW Berlin (Hochschule für Technik und Wirtschaft) besuchen Konferenzen und sind im Rahmen internationaler Projekte in aller Welt unterwegs. Eine Forschungsgruppe der HTW Berlin hat die Reisedaten des J ahres 2017 gesammelt, um die Verteilung der für die Dienstreisen gewählten Verkehrsmittel zu analysieren. Deren R ohdaten bilden die Ausgangslage für dieses Projekt, das die ausgestoßene Treibhausgasmenge der Verkehrsmittel Flugzeug, Auto, Reisebus und Zug miteinander vergleicht und visualisiert.

Ausgangswert bilden die ausgestoßenen Treibhausgase für einen Flug von Berlin nach München. Die meistgenutzte Verbindung der Angestellten im J ahre 2017. Dieser Wert bildet das Limit an Treibhausgasen, das den anderen Verkehrsmitteln zur Verfügung steht.

Die Strecke zwischen Berlin und München beträgt $528 \mathrm{~km}$ und stößt 10,6 kg Treibhausgase aus. Mit der gleichen Menge könnten im Auto $764 \mathrm{~km}$, im Reisebus $3317 \mathrm{~km}$ und im Zug 2948 km zurückgelegt werden. Der Unterschied ist deutlich zu erkennen. Mit der Treibhausgasmenge, welche bei einem Flug von Berlin nach München freigesetzt wird, würde der Zug bis nach Lissabon und der Reisebus tief ins Innere von Russland (z. B. Ufa) kommen.

Ziel ist es, diese Informationen in den Prozess der Planung und Buchung von Dienstreisen zu integrieren, um bei den Beteiligten das Problembewusstsein für $\mathrm{CO} 2$ sparende Verkehrsmittel zu wecken.
\end{abstract}

\section{Motivation}

Dienstreisen gehören für Hochschulmitglieder zum beruflichen Alltag. Zum wissenschaftlichen A ustausch ist der Besuch von internationalen $\mathrm{K}$ onferenzen unverzichtbar. Dazu kommen Projekte mit den entsprechenden
V erpflichtungen zu Dienstreisen anlässlich von M eetings und Präsentationen. Dies gilt auch für die A ngestell ten an der HTW Berlin (Hochschule für Technik und Wirtschaft). Sie unternehmen Dienstreisen in alle W elt. Diese Strecken werden häufig und wie selbstverständlich mit dem Flugzeug zurückgelegt. Laut den U mweltleitlinien der HTW B erlin sollen zwar öffentliche V erkehrsmittel vorgezogen werden, allerdings gilt dies nur, wenn es wirtschaftlich vertretbar ist. (HTW-Berlin, U mweltleitlinien, 2020) (HTW -B erlin, M obilität, 2019)

Eine Projektgruppe der HTW Berlin hat die Reisedaten des J ahres 2017 gesammelt und die V erteilung der V erkehrsmittel veranschaulicht (Fronk, Güccük, Höhne, M otuz, \& Zagorski, 2019). Deren Rohdaten bilden die A usgangslage für das vorliegende Paper.

Bei der Gegenüberstellung von verschiedenen Verkehrsmitteln wird meistens nur in den Kategorien Zeit und Kosten argumentiert. So bietet zum Beispiel der wohl am häufigsten genutzte Routenplaner von Google (Google, 2020) nur genau diese beiden Zielkriterien für eine Routenoptimierung an. Sicherlich sind Zeit und Strecke zwei wichtige und nachvollziehbare A rgumente, allerdings könnte die Gegenüberstellung noch um die A uswirkungen auf $\mathrm{K}$ lima und $\mathrm{G}$ esundheit erweitert werden, um auch die ökologische Dimension bei der Transportmittel wahl transparent zu machen.

Um A ngestellten, die ihre Geschäftsreisen mit alternativen $V$ erkehrsmitteln zurücklegen, eine A rgumentationshilfe an die $\mathrm{H}$ and zu geben, möchte dieses Projekt anhand einer $V$ isualisierung auf $K$ artenbasis den Unterschied zwischen Flugzeug einerseits und seinen A Iternativen beim Faktor Treibhausgase andererseits darstellen. Als zum Fliegen alternative V erkehrsmittel wurden das 
A uto, der Reisebus und der Zug gewählt. Bei denen es sich um gängige und leicht verfügbare $V$ erkehrsmittel handelt.

Ziel ist es, Informationen über diese A Iternativen in den Prozess der Planung und $B$ uchung von Dienstreisen zu integrieren, um bei den Beteiligten das Problembewusstsein für C O2-sparende M obilität zu wecken.

In den folgenden Abschnitten wird daher zunächst kurz das V isualisierungsverfahren der Erreichbarkeitsgraphen selbst erklärt. Darauf folgt die V orgehensweise zur Datenauswahl und -integration sowie die M öglichkeiten und Probleme der Nutzung der entsprechenden Tools unter ArcM ap. Dabei wird auch auf die Randbedingung der Studie eingegangen, besonders auf die Beschränkung auf open-source Datenmaterial. Erste Resultate und eine Diskussion der Schwierigkeiten bei der Durchführung beschließen das Paper.

\section{Methode}

Als Grundlage für das angewandte V isualisierungsverfahren dienen Erreichbarkeitsgrafen. Erreichbarkeitsgrafen werden in der K artografie angewandt, um darzustellen, welches $G$ ebiet bei vorgegebener Distanz von einem gegebenen Punkt aus erreicht werden kann. Zur Demonstration zeigt $A$ bbildung 1 als B eispiel die älteste bekannte Darstellung eines Erreichbarkeitsgrafen. A usgehend von London als zentralem Punkt und A usgangspunkt für die Entfernungsmessung zeigt sie die Reisezeit in die verschiedenen Regionen der W elt. Dabei wird ausgehend von London berechnet, wie viel Zeit auf einem gegebenen Wegenetz benötigt wird, die entsprechende Stelle auf dem Globus zu erreichen. Dargestellt ist dies in der historischen $\mathrm{K}$ arte durch in gewisser W eise aufeinander aufbauende, farblich gestaffelte Darstellungsschichten. Der dunkel grüne B ereich wird am schnellsten erreicht, gefolgt von einem hellgelben Ring und so weiter. (Galton, 2020)

A uch heute noch wird diese Technik angewandt, um zu visualisieren, welche Orte innerhalb einer gegebenen Zeit erreicht werden können. In dieser A rbeit wird als $\mathrm{Pa}-$ rameter für die Bestimmung der Ausdehnung der Erreichbarkeitszonen jedoch nicht das A ttribut "Zeit" angegeben vielmehr sollen die für die Reise benötigte E missionsmenge an Treibhausgas zugrunde liegen. Dazu soll zunächst eine Obergrenze an Emissionen für die zu fah- rende Strecke berechnet werden und anschließend angegeben und visualisiert werden, wie weit man auf einem gegebenen W egenetz unter Einhal tung dieses E missionsmaximums kommen kann. Der folgende A bschnitt erklärt die V orgehensweise im D etail.

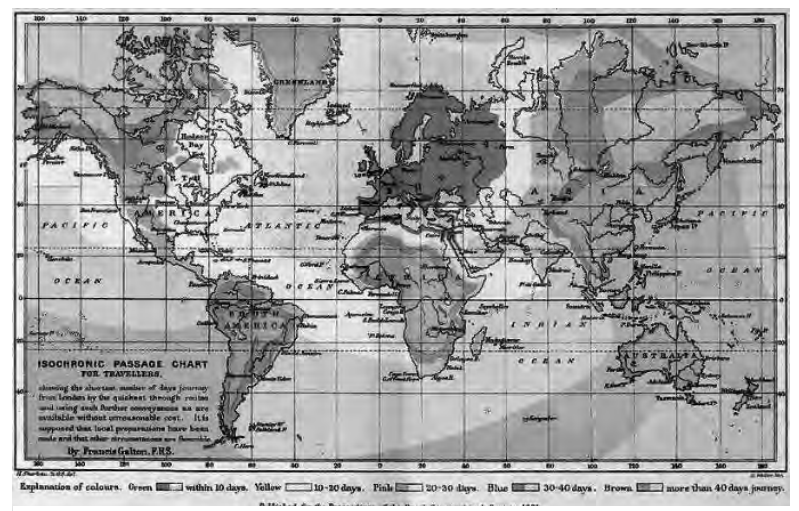

Figure 1: Die erste bekannte Erreichbarkeitskarte aus dem J ahre 1881. Sie zeigt die Reisezeit zu verschiedenen Regionen der Welt. (Galton, 2020)

\section{Vorgehensweise}

Nach der Veranschaulichung der M ethode folgt nun die Schilderung der einzelnen A rbeitsschritte. A Is Erstes wurde ein W egenetz benötigt. Als Zweites musste der Grenzwert bzw. M aximalwert an Emissionen berechnet werden, der den E rreichbarkeitsgrafen für die Transportmittel als $\mathrm{L}$ imit dienen soll. I m D ritten und letzten Schritt konnten die E rreichbarkeitsgrafen dann erstellt werden.

\subsection{Basisschicht erstellen}

Ein Erreichbarkeitsgraf berechnet die mögliche Strecke anhand eines gegebenen W egenetzes. Dieses Projekt vergl eicht die Flugstrecke mit den $V$ erkehrsmitteln $A$ uto, Reisebus und Zug, sodass zwei W egenetze benötigt werden. Einmal ein Straßennetz für $A$ uto und Reisebus und einmal ein Schienennetz für den Zug.

Als Basis dienen Daten des OpenStreetM ap-Projektes, die von den D ownloadservern der $\mathrm{G}$ eofabrik bezogen wurde (Geofabrik, 2019). Dabei wurden die europäischen Länder und Regionen als Shape-Files heruntergeIaden. A us diesen Shape-files wurden die für das Projekt benötigten Linienfeatures herausgesucht. Als Orientierung dienten die Wiki-Seiten des OpenStreetM ap-Projektes.

- Für das Straßennetz wurde der K ey "highway" mit den W erten "motorway", "motorway_link", 
"trunk", "trunk_link", "primary", "primary_link", "secondary", "second ary_link", "tertiary", "tertiary_link", "unclassi fied" verwendet.

- Für das Schienennetz wurde der K ey "railway" mit dem Wert "rail“ verwendet.

Ziel dabei war, bewusst auch niederrangige Verkehrswege aufzunehmen, aber andererseits dennoch die Datenmenge auf eine handhabbare Größe zu reduzieren. Die A uswahl der hier angeführten A ttributwerte ist für diesen Prototypen im Sinne einer $M$ achbarkeitsstudie sicher praktikabel. Für die Integration in das Zielsystem, das den gesamten Dienstreiseplanungsprozess umfassen soll, muss allerdings noch geprüft werden, ob sich die $M$ enge der notwendigen $W$ erte nicht weiterhin reduzieren lässt, ohne wesentliche A bstriche in der Genauigkeit der Ergebniskarte zu erzeugen. So könnte es sinnvoll sein, das zugrundeliegende Wegenetz in A bhängigkeit vom gewünschten $M$ aßstab der Zielkarte zu skalieren: $B$ ei einer weiteren Reise werden kleinste W egeverbindungen sicherlich weniger relevant für das Endergebnis sein als bei einer kürzeren Strecke.

$\mathrm{N}$ achdem die Datenmenge deutlich geschrumpft war, konnten die Länder und Regionen zu einer K arte zusammengefügt und in einer G eodatabase gespeichert werden, um die weitere V erarbeitung zu erleichtern.

\subsection{Berechnung der CO2 Faktoren}

Für jedes gewählte V erkehrsmittel wurde ein Grenzwert berechnet. D ieser $W$ ert berechnet sich aus dem A usgangswert und dem jeweiligen $V$ erbrauch pro Pkm (Personenkilometer). Als A usgangswert wird die ausgestoßene $M$ enge Treibhausgase für einen Flug von Berlin nach $M$ ünchen definiert. Dies war die meistgenutzte $V$ erbindung der HTW -Angestellten im J ahre 2017 (Fronk, Güccük, Höhne, M otuz, \& Zagorski, 2019). Die Flugstrecke von Berlin nach M ünchen beträgt $528 \mathrm{~km}$. Der Tabelle 1 wird die entsprechende Treibhausgasmenge pro $\mathrm{Pkm}$ entnommen und miteinander verrechnet.

E ine Person stößt demnach 10,6 kg T reibhausgase auf einem Flug von Berlin nach $M$ ünchen aus. A usgehend von diesem W ert konnte nun berechnet werden, welche Entfernung andere Transportmittel mit dem durch den Flugverkehr vorgegebenen Emissionsmaximum zurücklegen könnten.

Tabelle 1 enthält die entsprechend berechneten Distanzen, die als Grenzwert für das jeweilige V erkehrsmit- tel eingetragen wurden. Basis waren dabei die inzwischen allgemein anerkannten durchschnittlichen E missionen gemäß der Zusammenstellung des U mweltbundesamtes (U mweltbundesamt, 2020). Die V erwendung dieser Durchschnittswerte wird in einem folgenden $A b$ schnitt diskutiert werden.

Tabelle 1: Durchschnittliche Emissionen der Verkehrsmittel im J ahre 2017. (U mweltbundesamt, 2020)

\begin{tabular}{|l|c|c|c|}
\hline $\begin{array}{l}\text { Verkehrs- } \\
\text { mittel }\end{array}$ & $\begin{array}{l}\text { Treibhaus- } \\
\text { gas } \\
\text { (g/Pkm) }\end{array}$ & A uslastung & $\begin{array}{l}\text { Distanz } \\
\text { (km) }\end{array}$ \\
\hline Flugzeug & 201 & $82 \%$ & 528 \\
\hline A uto & 139 & $\begin{array}{l}1,5 \text { Pers. } \\
\text { pro PK W }\end{array}$ & 764 \\
\hline Reisebus & 32 & $60 \%$ & 3317 \\
\hline $\begin{array}{l}\text { Zug } \\
\text { (Fern- } \\
\text { verkehr) }\end{array}$ & 36 & $56 \%$ & 2948 \\
\hline
\end{tabular}

\subsection{Distanzgrafen erstellen}

$N$ ach der B erechnung der G renzwerte für die erreichbare Distanz und der erfolgreichen Erstellung des Streckennetzes, konnte damit begonnen werden die Distanzgrafen zu berechnen. Dazu wurde die Erweiterung „A rcG is N etwork A nalyst" von A rcG is verwendet.

Der "ArcGis Network A nalyst" benötigt ein N etzwerk-Dataset, das aus miteinander verbundenen $K$ anten (Linien) und Verbindungsknoten (Punkten) besteht. In diesem Projekt wird dazu einmal das Straßennetz und einmal das Schienennetz in ein N etzwerk-Dataset umgewandelt, um danach eine Netzwerkanalyse durchführen zu können.

B ei der Analyse können, wie bereits geschrieben, zeitliche Faktoren sowie Distanzen angegeben werden. Dieses Projekt arbeitet mit vorgegebenen Distanzen. Als Distanz wird für das jeweilige Verkehrsmittel der Wert in der Spal te Distanz in Tabelle 1 entnommen.

A ufgrund von der erheblichen Datenmenge konnten in A nbetracht der zur V erfügung stehenden Zielplattform, keine Polygone für den Reisebus und den Zug erstellt werden. Diese wurden nachträglich mittels des Tools "Feature in Polygon" aus den Liniennetzen erzeugt. 


\section{Ergebnisse und Diskussion}

\subsection{Erreichbarkeitsgraphen}

Die A bbildungen 2, 3 und 4 stellen den jeweiligen $\mathrm{Er}$ reichbarkeitsgrafen für die Verkehrsmittel A uto, Reisebus und Zug dar. Die A bbildungen 3 und 4 zeigen insbesondere, dass sich die mögliche W egstrecke stark erweitert, wenn für die Reise der Reisebus oder der Zug gewählt wird. Dagegen fällt der Unterschied zwischen PKW und Flugzeug erstaunlich gering aus. (A bb. 2)

W ährend des Projektes gab es einige Faktoren, die die Qualität der V isualisierung wesentlich beeinflusst haben. $D$ as war zum einen die bereits genannte $B$ eschränkung in der Rechenleistung, andererseits die Q ualität des zugrundeliegenden W egenetzes, sowie schließlich auch die verwendeten pauschalierten Treibhausgaswerte. A uf diese Punkte soll im Folgenden eingegangen werden.

\subsection{Problem Rechenleistung}

Die genutzten Rechner stießen an die Grenzen ihrer Berechnungsmöglichkeiten für den "Network A nalysten“. Es war nicht in vertretbarer Zeit möglich, ein Polygone für den Zug und den Reisebus zu berechnen. Die Berechnung eines Erreichbarkeitsgraphen wurde auf einen Standard-PC nach etwa 36 Stunden Rechenzeit abgebrochen. Stattdessen musste ein U mweg über die berechneten $L$ inien gegangen werden, was eine potenzielle Fehlerquelle ist, da bei dieser $\mathrm{V}$ orgehensweise einige B ereiche nicht korrekt dargestellt werden. Das betrifft zum B eispiel B ereiche in Norwegen und Schweden.

Tatsächlich liegen aber in den nicht berücksichtigten Zwischenräumen zwischen den durch das Liniennetz erreichbaren Punkten ja gerade keine $V$ erkehrswege, die es ermöglichen würden, in diese Z wischenräume vorzudringen. Die Polygonbildung glättet lediglich den Rand des Ergebnisses der Erreichbarkeitsrechnung. B esonders in Bezug auf die Genauigkeitsanforderungen des Prototypen stellt dies keine wesentliche E inschränkung dar.

\subsection{Problem Basis-Geodatendaten}

Das A usgangsmaterial basiert auf Ländern, die Geofabrik dem europäischen Raum zuordnet. So werden $\mathrm{Ge}$ orgien, Russland (der nicht europäische Teil) und die Türkei zu Europa zugerechnet, A serbaidschan oder Kasachstan jedoch nicht. Diese $\mathrm{G}$ renzziehung durch die $\mathrm{Ge}$ ofabrik ist dabei nicht ganz klar und wird auch auf den Seiten des A nbieters nicht näher erläutert (Geofabrik, 2019).

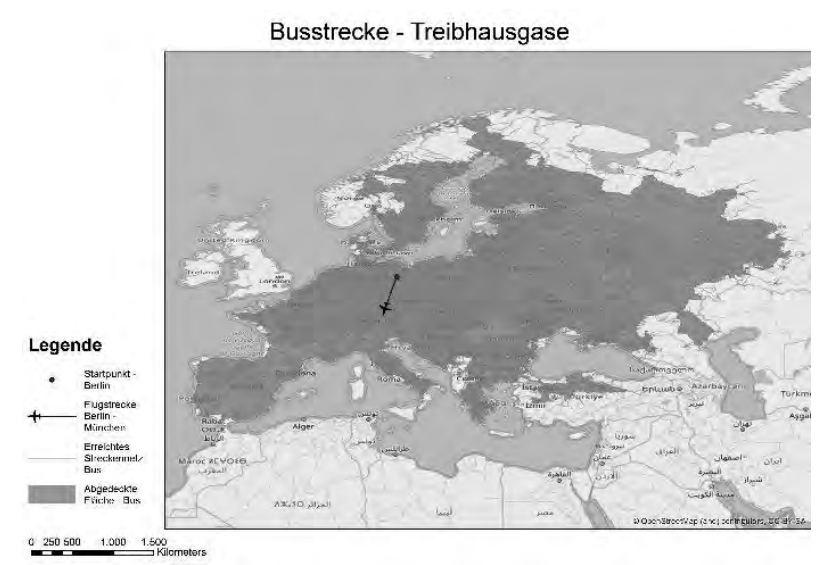

Figure 2: Erreichbarkeitsgraf für einen PKW, wenn 10,6 kg Treibhausgase zur Verfügung stehen.

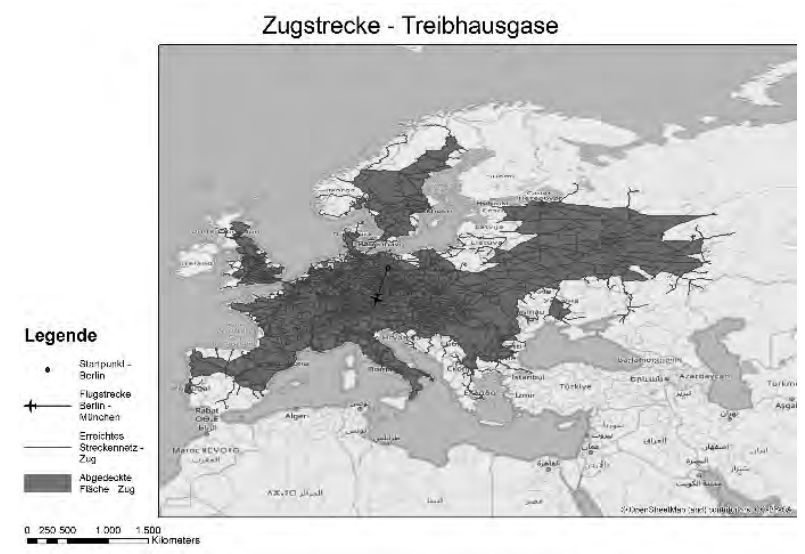

Figure 3: Erreichbarkeitsgraf für einen Reisebus, wenn 10,6 kg Treibhausgase zur Verfügung stehen

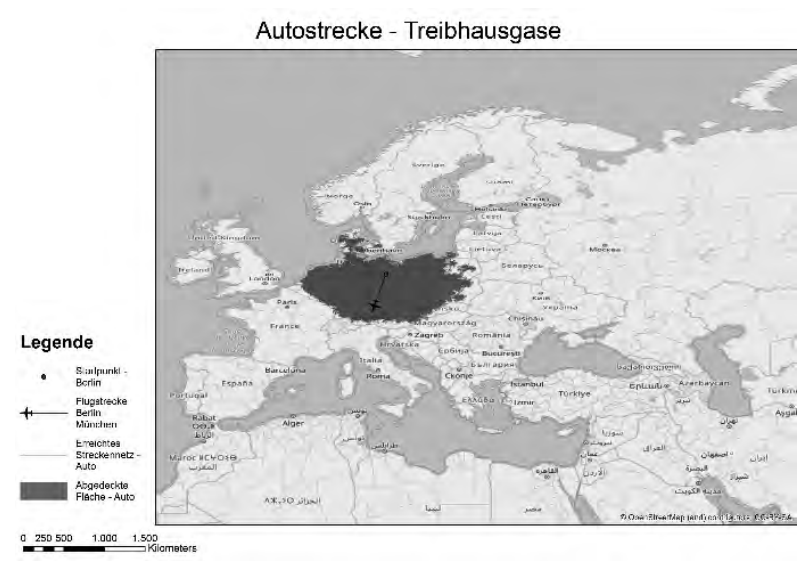

Figure 4: Erreichbarkeitsgraf für einen Zug, wenn 10,6 kg Treibhausgase zur Verfügung stehen. 
Die Auswahl der hier berücksichtigten Länder erscheint somit recht willkürlich. Bei nachfolgenden A nwendungen sollten daher die ungefähr benötigten R eichweiten vorab abgeschätzt werden und als R ichtwert dienen, welche Länder bei der Erstellung der Basisschicht berücksichtigt werden sollten.

Ein auffälliger Unterschied zwischen Abbildung 3 und 4 ist, dass es eine V erbindung zwischen Frankreich und G roßbritannien für das Schienennetz gibt, aber nicht für das Straßennetz. Sodass die Reisebuskarte nicht die theoretisch mögliche Strecke bis nach Großbritannien abbildet. U rsache hierfür ist, dass der Reisebus nur mittels des Zuges den Tunnel oder mittels einer Fähre den $K$ anal durch-/überqueren könnte. Der Reisebus muss selbst auf andere Verkehrsmittel ausweichen. Da keine Straße den Ärmelkanal durchquert, kann der „Network A nalyst" diese Strecke nicht ohne W eiteres berücksichtigen. Hier könnte man im Einzelfall durch eine individuelle Nachbearbeitung des Streckennetzes abhelfen, ein A ufwand der für den Prototypen nicht getrieben wurde.

\subsection{Problem Emissionswerte für die jeweiligen Verkehrsmittel}

Die gewählten W erte beruhen auf Durchschnittswerten herausgegeben vom U mweltbundesamt. Die tatsächlich ausgestoßene Menge an Treibhausgasen für einen Flug zwischen Berlin und $M$ ünchen könnte höher sein, weil während des Starts und der Landung tendenziell mehr Treibhausgase ausgestoßen werden, als wenn die Reiseflughöhe erreicht ist. Daher haben Kurzstreckenflüge (unter $750 \mathrm{~km}$ ) im Schnitt einen höheren T reibhausgasausstoß als Langstreckenflüge. Bei der Tabelle des U mweltbundesamtes ist allerdings nur ein W ert für das Verkehrsmittel Flugzeug angegeben. (Umweltbundesamt, 2020) Bei der Übertragung auf sämtliche Dienstreisen sollte daher unbedingt eine entsprechende Differenzierung gemäß der $L$ änge des Fluges bzw. gemäß einer detaillierteren K lassifizierung des Fluges vorgenommen werden. Für die $M$ achbarkeitsstudie des hier vorgestel Iten Prototyps wurde zunächst auf diese Präzisierung verzichtet, zumal bei den Reisen der Datenbasis auch nicht abgefragt wurde, ob es sich bei der geflogenen $V$ erbindung um einen Direktflug mit oder ohne Zwischenlandung handelte.

Des Weiteren kann die A uslastung eines PK W s bei einer tatsächlichen Dienstreise höher sein, als die hier angegebenen 1,5 Personen pro PKW. Die Reichweite des F ahrzeugs erhöht sich, umso mehr Personen im Fahrzeug sitzen. Dies ist allerdings eine Information, die im betrachteten U se-Case bei der Reiseplanung einer konkreten Dienstreise durchaus zur V erfügung steht und als zusätzlicher Parameter vom Benutzer abgefragt werden könnte. Damit kann dann ein für die aktuell untersuchte Dienstreise spezifischer E missions-W ert und damit eine spezifische potenzielle Reichweite leicht ermittelt werden.

\section{$5 \quad$ Fazit und Ausblick}

Trotz der genannten Einschränkungen ist der Unterschied der möglichen Strecke zwischen Flugzeug und anderen V erkehrsmitteln, speziell zum Reisebus und Zug, deutlich zu erkennen. M it der Treibhausgasmenge, welche bei einem Flug von B erlin nach $M$ ünchen freigesetzt wird, würde der Zug bis nach Lissabon und der Reisebus bis weit hinter M oskau ins I nnere von R ussland kommen. Dieses Projekt möchte niemanden dazu auffordern, statt mit dem Flugzeug nach $M$ ünchen, lieber mit dem $B$ us nach Lissabon zu fahren. Allerdings haben erste Präsentationen im Rahmen der Hochschule gezeigt, dass diese A rt der $V$ isualisierung durchaus das Problembewusstsein fördert, indem es anschaulich aufzeigt, welches Einsparpotenzial an Treibhausgasen bei dem V erzicht auf Flugreisen vorhanden ist.

Im W eiteren ist daher geplant, eine im Funktionsumfang reduzierte, jedoch für die jeweils aktuell zu planende Dienstreise individuell parametrisierbare Version zu erstellen und diese Version standardmäßig bei jeder B eantragung einer Flugreise zur V erfügung zu stellen.

\section{Literaturverzeichnis}

Fronk, M., Güccük, A., Höhne, M., M otuz, A., \& Zagorski, A . (2019). Erfassung und A uswertung der mit Dienstreisen verbundenen U mweltauswirkungen der HTW Berlin. In J. Wittmann, Simulation in den Umwelt- und Geowissenschaften, Workshop Kassel 2019 (S. 15-26). Shaker: Aachen.

Galton, F. (31.. 03. 2020). Wikipedia. Von https://en.wikipedia.org/wiki/File:I sochronic P assage_Chart_Francis_Galton_1881.jpg abgerufen 
Geofabrik. (09.. 07. 2019). O penStreetM ap Europe. V on https://download.geofabrik.de/europe.html. abgerufen

HTW-Berlin. (09.. 07. 2019). Mobilität. Von htwberlin.de/einrichtungen/zentralehochschulverwaltung/technischedienste/organisationatd/umweltmanagement/aktivitaeten-undtipps/mobilitaet/ abgerufen

HTW-Berlin. (31.. 03. 2020). U mweltleitlinien. Von https://www.htwberlin.de/fileadmin/HTW/Zentral/ZHV_IIQM _Qualitaetsmanagement/08_U mweltleitlinien_f inal.pdf. abgerufen

U mweltbundesamt. (31.. 03. 2020). E missionsdaten. V on https://web.archive.org/web/20190718134549/ https://www.umweltbundesamt.de/themen/verk ehr-laerm/emissionsdaten abgerufen 